\title{
Finite Element Analyses and Experimental Study about Normal and Low Temperature Properties of Cryogenic COPV
}

\author{
Dao-Lian WANG ${ }^{1, a}$, Chuan-Jun LIAO ${ }^{2, b, *}$, Bin SITU², Jing LIU² \\ ${ }^{1}$ School of Aeronautics, Northwestern Polytechnical University, Xian 710049, China \\ ${ }^{2}$ Beijing Institute of Astronautical Systems Engineering, Beijing 100076, China \\ a13341188559@163.com, ${ }^{b}$ liaochuanjunl@163.com \\ * Corresponding author
}

Keywords: Cryogenic, COPV, Finite element, Normal temperature, Low temperature.

\begin{abstract}
Based on the ANSYS software, a finite element model using for analyzing the properties of the developed cryogenic composite overwrapped pressure vessels (COPV) is established. The stress and strain conditions under the operational pressure were analyzed including of the overwrapped fibrous layer and the metal inside lining, which were used to respectively explain the properties under the normal temperature and the low temperature. The differences between the normal temperature properties and the low temperature properties were also clarified. Simultaneously, the weakness of the cryogenic COPV was identified. A test apparatus applying for the experimental studies of the developed cryogenic COPV was build, the pressure-circulation experiments under the normal temperature and the temperature of liquid nitrogen were respectively carried out, and the actual service requirements of the developed cryogenic COPV were tested.
\end{abstract}

\section{Instructions}

The composite overwrapped pressure vessels (COPV) is manufactured by enwinding high strength fibers on metal or non-metal inside linings, and have many merits including of light weight, good rigidity, high reliability, long service time, good fatigue resistance etc. The COPV have been comprehensively applied for the aviation, space and other industries ${ }^{[1]}$. The resin of the COPV is generally the ethoxyline resin that has many merits including of good mechanical property, strong adhesion, ease solidification etc, but its plasticity and toughness will decrease with the descending of the ambient temperature, so mainly is used for normal conditions ${ }^{[2-5]}$. For satisfying the application needs of low temperature conditions for example liquid hydrogen and liquid oxygen, many countries are developing the cryogenic COPV, and many related key techniques have been solved ${ }^{[6-7]}$. At the present time, the cryogenic COPV has some practical applications.

The working performances of the cryogenic COPV should be clarified, which is the basic requirement of its development and applications. Using the finite element method, the normal and low temperature properties of the developed cryogenic COPV were analyzed in this paper, and were tested by the practical working conditions.

\section{Structure of Cryogenic COPV}

The developed cryogenic COPV is constituted by the metal inside lining and the strengthen structure of fiber winding layer, and its volume is $56 \mathrm{~L}$ and its picture is shown in Fig. 1 . The metal inside lining composes of the cylindrical section, spheroidicity end plate, field joint and porting joint etc. The fiber winding layer is fabricated by the resin base and the strengthen fiber.

\section{Simulation Analyses}

\subsection{Finite Element Model}

Based on the ANSYS software, the finite element analysis of the 56L cryogenic COPV is performed. According to the structure and load characteristics, the 1/8 finite element model is developed, as 
shown in Fig. 2. The analysis of the metal inside lining and the fiber layer respectively adopts the solid 95 element and the shell 91 element. The contact of the metal inside lining with the fiber layer is simulated by the contact of the deformable body with the deformable body.

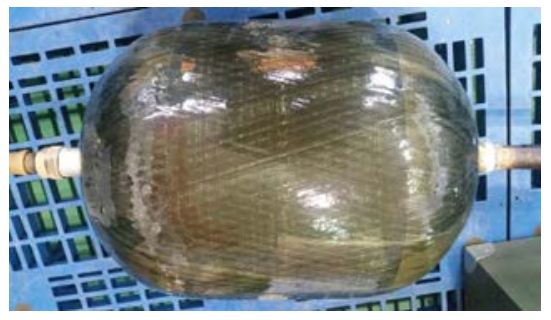

Fig. 1 Picture of the $56 \mathrm{~L}$ cryogenic COPV

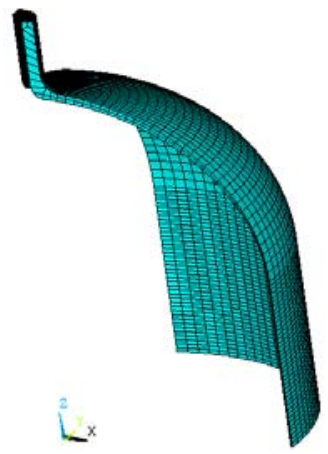

Fig. 2 Finite element model of the cryogenic COPV

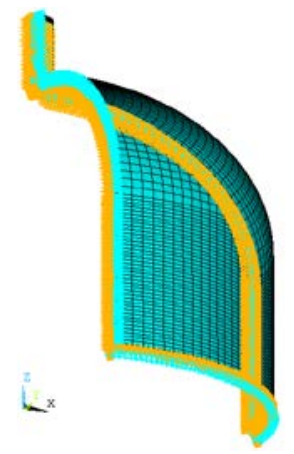

Fig. 3 Boundary conditions of the finite element model

The symmetry boundary is adopted in the finite element model, as shown in Fig. 3. All the freedoms of the end surface of the nozzle are constrained.

\subsection{Analysis of Normal Temperature Property}

Under the operational pressure (35 MPa) of normal temperature (293 K), the Mises stress and the Mises strain of the metal inside line are respectively shown in Fig. 4 and Fig. 5, and the following results can be obtained. The maximum Mises stress is $304 \mathrm{MPa}$ that is slightly bigger than the yield limit $300 \mathrm{MPa}$ of the material, and is less than the breaking point of $320 \mathrm{MPa}$ of the material. The maximum Mises strain is 0.032 , which is far less than the breaking strain 0.12 of the material. Therefore, it can be concluded that the metal inside line doesn't damage and can service normally under the operational pressure of normal temperature.

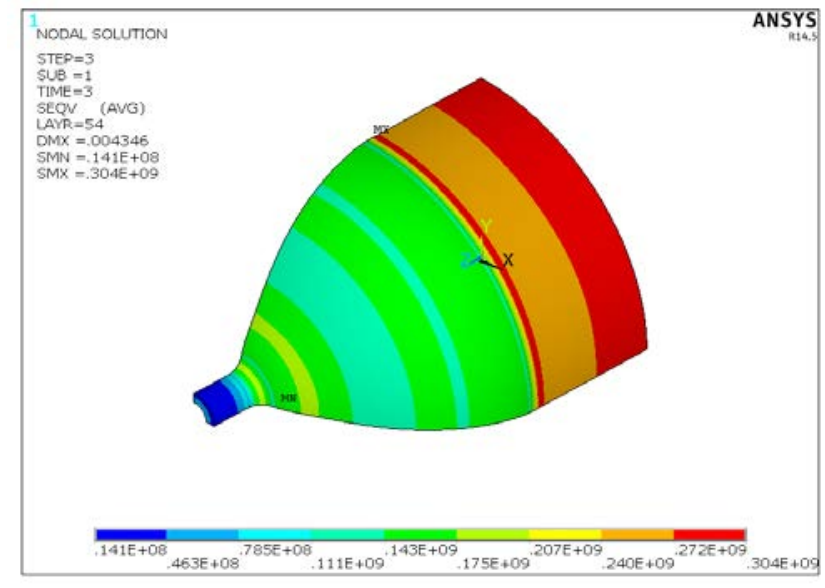

Fig. 4 Mises stress cloud image of the metal inside lining

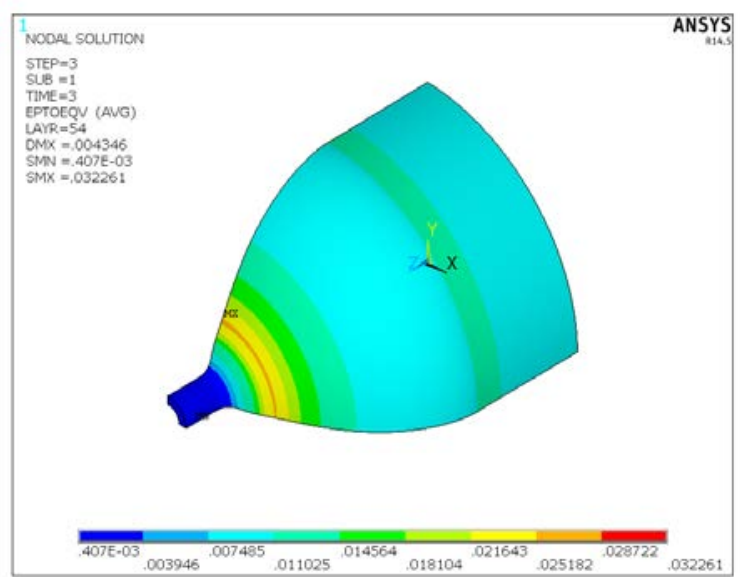

Fig. 5 Mises strain cloud image of the metal inside lining

For the inner surface of the metal inside line, the Mises stress path and the Mises strain path are respectively shown in Fig. 6 and Fig. 7. The selected path is the bus bar of the inner surface of the metal inside line, which is from the end plate of the bottleneck to the middle of the straight barrel shell. It can be found from Fig. 6 that the maximum Mises stress is $294.3 \mathrm{MPa}$, and locates at the middle point of the straight barrel shell and the joint point between the end plate and the straight barrel shell. The Mises stress of the end plate segment is uniform, and is about $165 \mathrm{MPa}$ that is 
lower than the yield limit $300 \mathrm{MPa}$ of the material. The Mises stress of the joint point between the end plate and the straight barrel shell generates mutation and the stress increments suddenly. It can be found from Fig. 7 that the maximum Mises strain is 0.026, and far less than the breaking strain 0.12 , and is near the bottleneck of the end plate. The Mises strain of the straight barrel shell is homogeneous and is about 0.011 , from which it can be concluded that the metal inside lining doesn't break. The maximum Mises stress in Fig. 4 locates at the outside surface of the metal inside lining, and the maximum Mises stress in Fig. 6 locates at the inside surface of the metal inside lining. The former is slightly larger than the latter, which is caused by the contact of the outside surface with the fibrous layer.

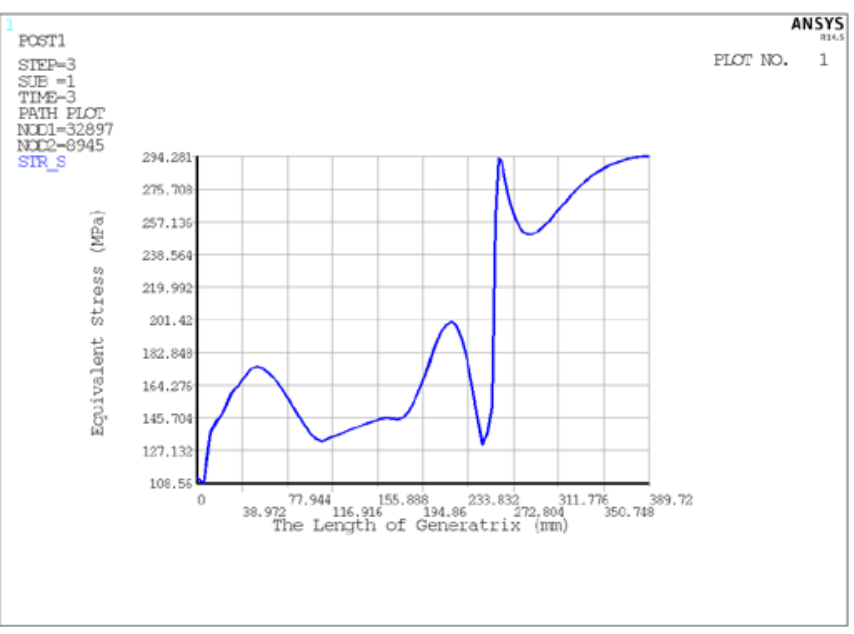

Fig. 6 Mises stress path of the inner surface of the metal inside lining

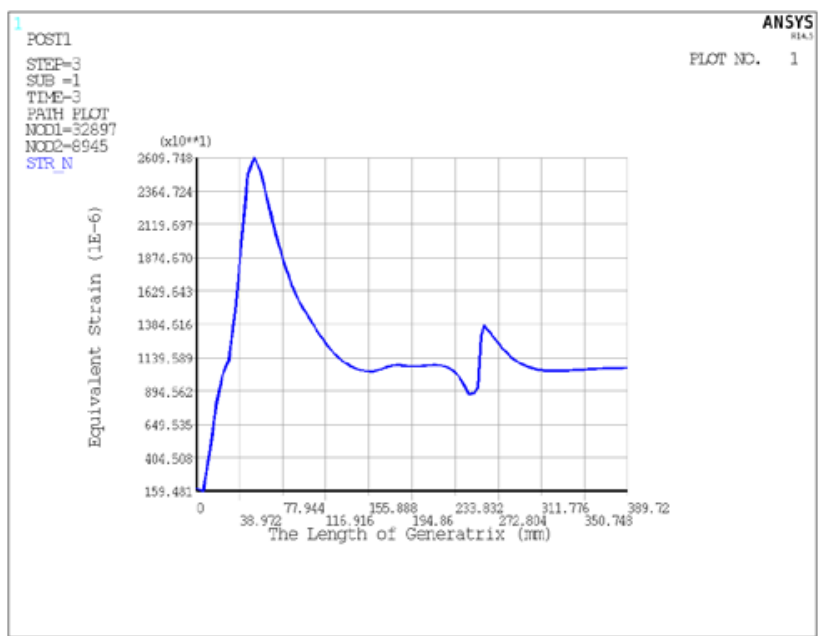

Fig. 7 the Mises strain path of the inner surface of the metal inside lining

For the outer layer of the overwrapped fibrous layer, the stress and the stain long the bus bar are respectively shown in Fig. 8 and Fig. 9, from which it can be found that there are individually two peak values for both the stress diagram and the strain diagram, and respectively located at the ending of the nozzle and the joint point between the end plate and the straight barrel segment. For the straight barrel segment, both the stress distribution and the strain distribution are uniform. For the practical winding course of the cryogenic COPV, there are fiber accumulations at the ending of the nozzle, so it is difficult to accurately analyze the stress and the strain. Therefore, the strength at the ending of the nozzle is guaranteed by the metal inside lining, and the results can be ignored when analyzing the overwrapped fibrous layer. From Fig. 8, it can be found that the axial stress at the joint point between the end plate and the straight barrel segment is maximal. The value is about $1360 \mathrm{MPa}$ that is lower than the 1-direction tensile-strength, but is higher than the tensile-strengths of both the 2-direction and the 3-direction. Therefore, there might happen matrix crack, but don't happen fiber break, and the use of the cryogenic COPV can be ensured. From Fig. 9, it can be found that the radial strain at the joint point between the end plate and the straight barrel segment is maximal, and the value is about 0.0105. Based on mentioned above, it can be concluded that the joint point between the end plate and the straight barrel segment is the weakest area of the cryogenic COPV, and the position might be here if the cryogenic COPV happen strength failure. 


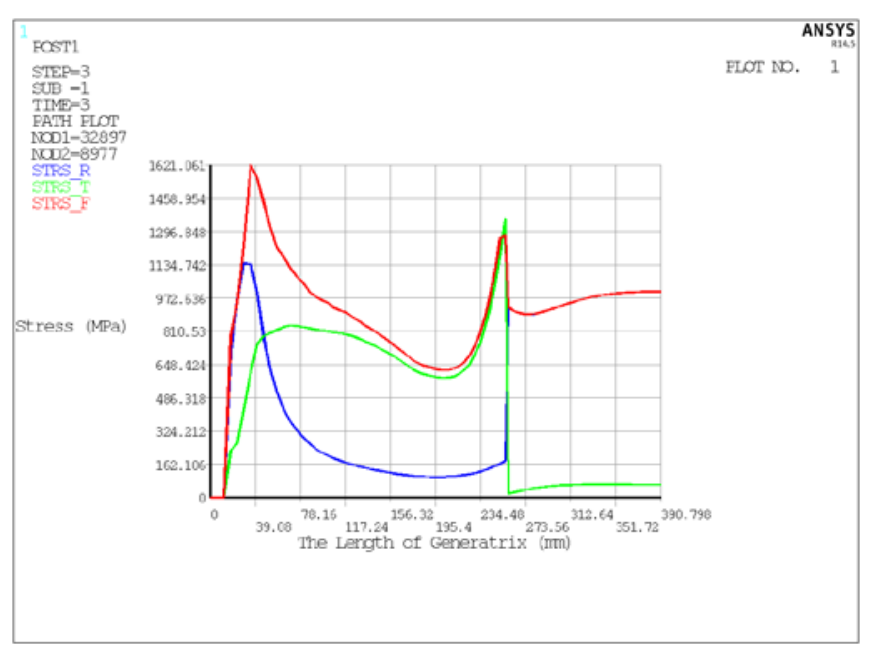

STRS_R—circle, STRS_T—radial, STRS_F-axial

Fig. 8 Stress path of the outer layer of the overwrapped fibrous layer

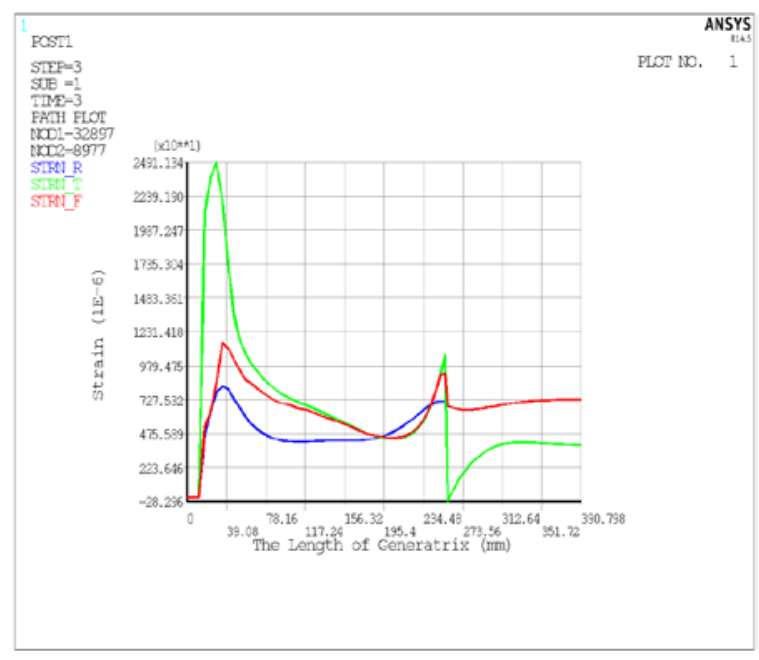

Fig. 9 Strain path of the outer layer of the overwrapped fibrous layer

\subsection{Analysis of Low Temperature Property}

Under the operational pressure (35 MPa) of low temperature $(90 \mathrm{~K})$, the Mises stress and the Mises strain of the metal inside line are respectively shown in Fig. 10 and Fig. 11, and the following results can be obtained. The maximum Mises stress is $361 \mathrm{MPa}$ that is slightly bigger than the yield limit $345 \mathrm{MPa}$ of the material, and is less than the breaking point of $450 \mathrm{MPa}$ of the material. The maximum Mises strain is 0.018 , which is far less than the breaking strain 0.18 of the material. Therefore, it can be concluded that the metal inside line can service normally under the operational pressure of low temperature.

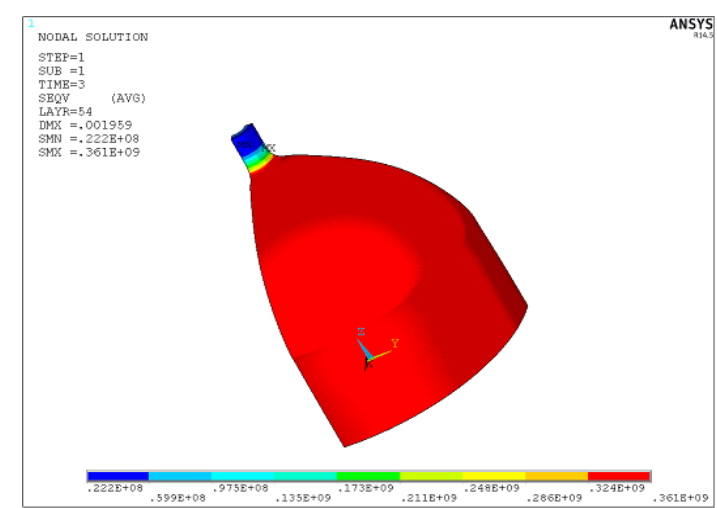

Fig. 10 Mises stress cloud image of the metal inside lining

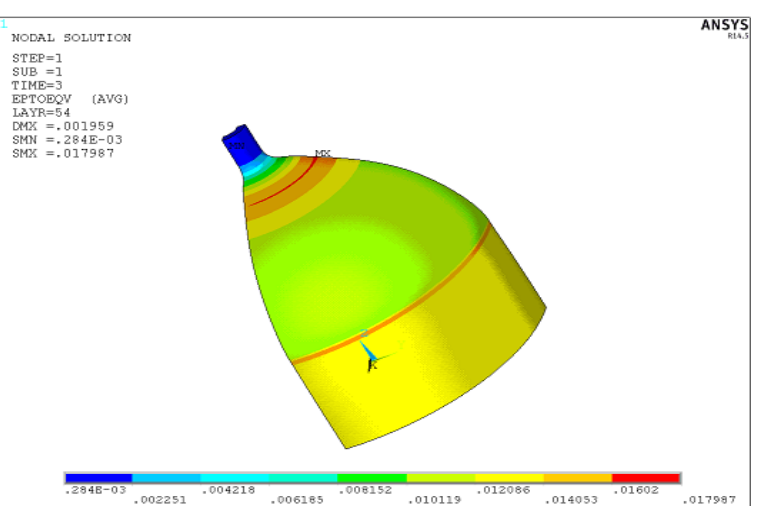

Fig. 11 Mises strain cloud image of the metal inside lining

For the inner surface of the metal inside line, the Mises stress path and the Mises strain path are respectively shown in Fig. 12 and Fig. 13. The selected path is the same bus bar of the inner surface of the metal inside line. Both the maximum stress and the maximum strain are found to be at the end plate near the nozzle, and the stress and the strain of the joint point between the end plate and the straight barrel shell are also large. Comparing the analytical results of the normal temperature with that of the low temperature, the following conclusions can be obtained. The stress level of the metal inside lining under low temperature is higher, which is caused by the improvements of both the yield limit and the breaking point of 6061 alloy under the low temperature. Under the low 
temperature, the stress distribution of the metal inside lining is more homogeneous. The positions of the maximum strain are different, and are at the end plate near the nozzle under low temperature, but are at the joint point between the end plate and the straight barrel shell under normal temperature, which is caused by that the coefficient of linear expansion of the material of the metal inside lining doesn't match with that of the composite.

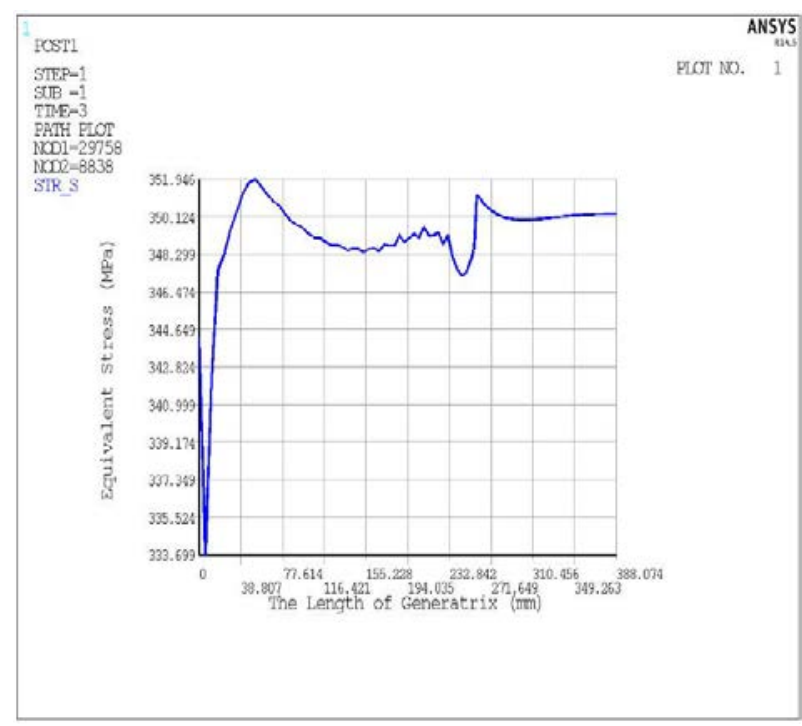

Fig. 12 Mises stress path of the inner surface of the metal inside lining

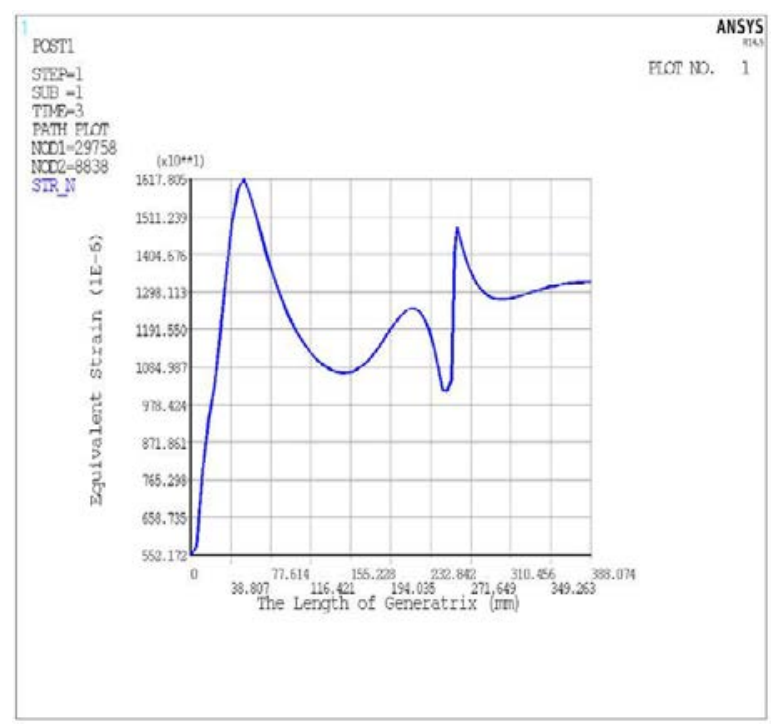

Fig. 13 Mises strain path of the inner surface of the metal inside lining

For the outer layer of the overwrapped fibrous layer, the stress and the stain long the bus bar are respectively shown in Fig. 14 and Fig. 15, from which it can be found that there are individually two peak values for both the stress diagram and the strain diagram, and respectively located at the ending of the nozzle and the joint point between the end plate and the straight barrel shell. For the straight barrel segment, both the stress distribution and the strain distribution are uniform. The results mentioned above are similar with that under the normal temperature, but the stress level and the strain level at the end plate and the joint decrease under low temperature. If the stress and the strain at the ending of the nozzle are ignored, the circumferential stress and strain at the joint point between the end plate and the straight barrel segment are maximal. The maximum stress is about $827 \mathrm{MPa}$, and the maximum strain is about 0.008 . The maximum stress is lower than the 1-direction tensile-strength, but is higher than the tensile-strengths of both the 2-direction and the 3-direction. Therefore, there might happen matrix crack, but don't happen fiber break, and the use of the cryogenic COPV can be ensured. It can be concluded that the joint point between the end plate and the straight barrel segment is the weakest area of the cryogenic COPV, and the position might be here if the cryogenic COPV happen strength failure. Comparing the analytical results of the normal temperature with that of the low temperature, it can be found that both the stress and the strain of the outermost layer of the composite layer decrease under the low temperature, which is caused by the shrinkage of the materials under low temperature. 


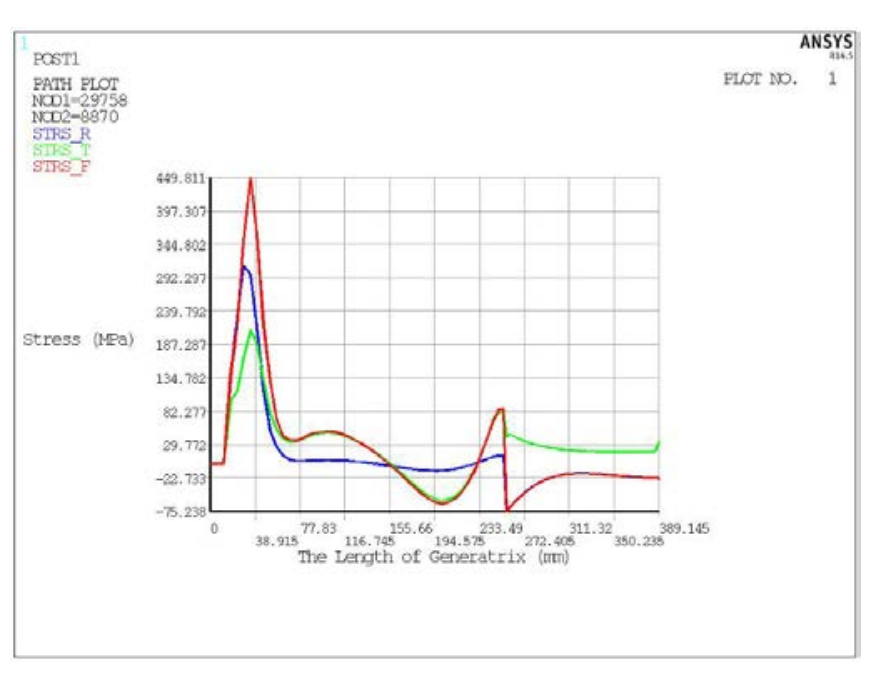

Fig. 14 Stress path of the outer layer of the overwrapped fibrous layer

\section{Experimental Studies}

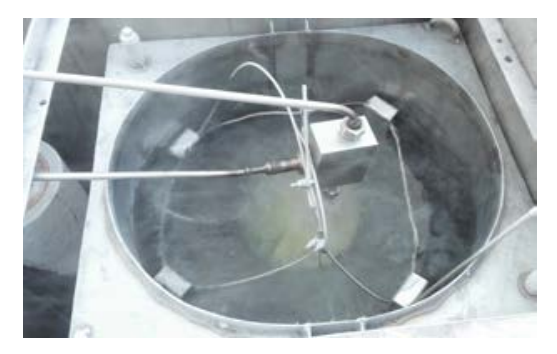

Fig. 16 Test apparatus of pressure- circulation experiment under the temperature of liquid nitrogen

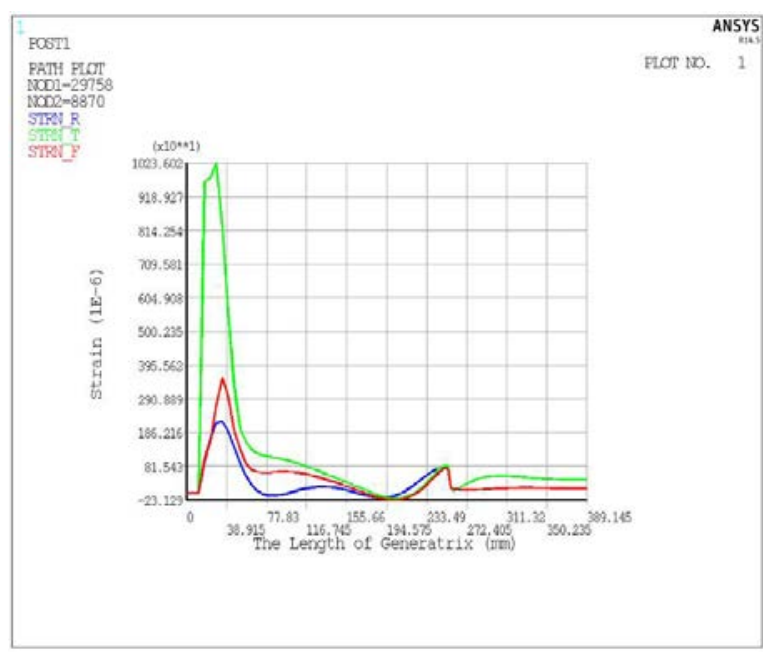

Fig. 15 Strain path of the outer layer of the overwrapped fibrous layer

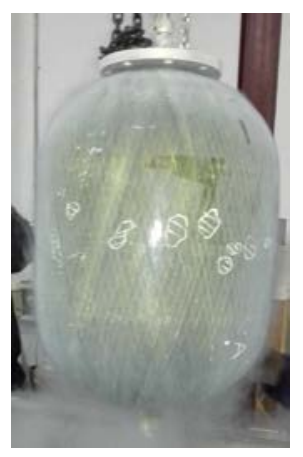

Fig. 17 Picture of the tested sample taken out from the test apparatus after the experiment

For the developed cryogenic COPV, the pressure-circulation experiment of one hundred times of $0 \sim 35 \mathrm{MPa} \sim 0$ was carried out Under the normal temperature, and the testing medium is water. The maximum test pressure is same with that of the simulated analysis. In the experimental course, uncommon conditions such as leakage and breaking don't happen, and the experimental results meet the requirements. Secondly, the pressure- circulation experiment of twenty-five times of $4 \mathrm{MPa} 35 \mathrm{MPa} 4 \mathrm{MPa}$ was carried out under the temperature of liquid nitrogen, and the test apparatus is as shown in Fig 16. After the experiment, the test sample was taken out from the test apparatus, as shown in Fig. 17. By the detections, the status of the test sample is good, and no abnormal conditions happened. So the normal and low temperature prosperities were verified, and were consistent with the results of the simulation analysis.

\section{Conclusions}

In order to analyze and verify the normal and low temperature properties of the developed the cryogenic COPV, the analytical and experimental works are carried out, and the main conclusions are as follows. The finite element analysis model based on the ANASYS software was developed to study the cryogenic COPV, and both the normal temperature property and the low temperature property can be obtained. By analyzing the stress and strain conditions of both the overwrapped 
fibrous layer and the metal inside lining, not only the stress and deformation features of the cryogenic COPV can be obtained under the operational pressure, but also the weakness of cryogenic COPV can be identified simultaneously. The test apparatus applying for the experimental studies of the cryogenic COPV was build, and the properties under the normal temperature and the low temperature were tested. The actual service requirements of the developed cryogenic COPV were satisfied.

\section{References}

[1] G. Feng, K.J. Xu, C.G. Zhou, Structure performance and application of composite cylinders, Engineering Plastics Application. 39(2011)50-52.

[2] G.Q. Liu, D.F. Yang, J.M. Zhai. Approximate formula of bullet proof velocity for high strength GFRP, Fiber Composites, 26(2001)11-12.

[3] L.G. Zhang, Z.G. Zhang. Analysis of defects in advanced composites, Fiber Reinforced Plastics/Composite. 23(2001)42-45.

[4] B. Yu, ZH.D. Liu, W.W. Zhao, et al, Development of world-wide composite gas cylinder and analysis of chinese COPV standard (-), Pressure Vessel Technology. 28(2012)47-52.

[5] K. Liu, R.SH. Wang, Y.M. Shi, et al, Cryogenic performances of fibre reinforced polymer matrix composites, Cryogenics. 35(2006)35-44.

[6] D.A. Norman, R.E. Robertson, Rigid-particle toughening of glassy polymers, Polymer. 44(2002) 2351-2362.

[7] Y. Morino, T. Shimoda, T. Morimoto, et al, Applicability of CFRP materials to the cryogenic propellant tank for reusable launch vehicle (RLV), Advanced Composite Materials. 10(2001)339-347. 